Expressive Genetic Programming: Concepts and Applications

Lee Spector Nicholas Freitag McPhee Cognitive Science, Hampshire College Division of Science \& Mathematics $\begin{array}{cc}\text { Computer Science, UMass } & \text { University of Minnesota, Morris } \\ \text { Amherst, MA USA } & \text { Morris, Minnesota USA }\end{array}$ Amherst, MA USA mcphee@morris.umn.edu

\section{Instructors (1)}

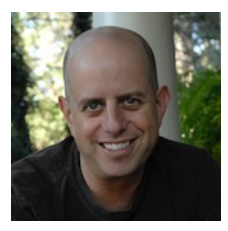

Lee Spector is a Professor of Computer Science in the School of Cognitive Science at Hampshire College in Amherst,

Massachusetts, and an adjunct professor in the Department of

Computer Science at the University of Massachusetts, Amherst He received B B in Philosophy from Oberlin College in 1984 and a Ph.D. from the Department of Computer Science at the University of Maryland in 1092. His areas of teaching and research includy genetic and evolutionary computation quantum computation, and variety of intersections between computer science, cognitive, and science, evolulion biology, and the arts. He is the Editorin-Chief of the jurnal Genetic Programming and Evolvable Mahines (published by Springer) and a member of the editorial board of (pvolutionar Compu) Evolutionary Computation (published by MIT Press). He is also a

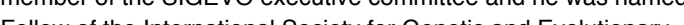
Fellow of the International Society for Genetic and Evolutionary

More info: http://hampshire.edu/lspector

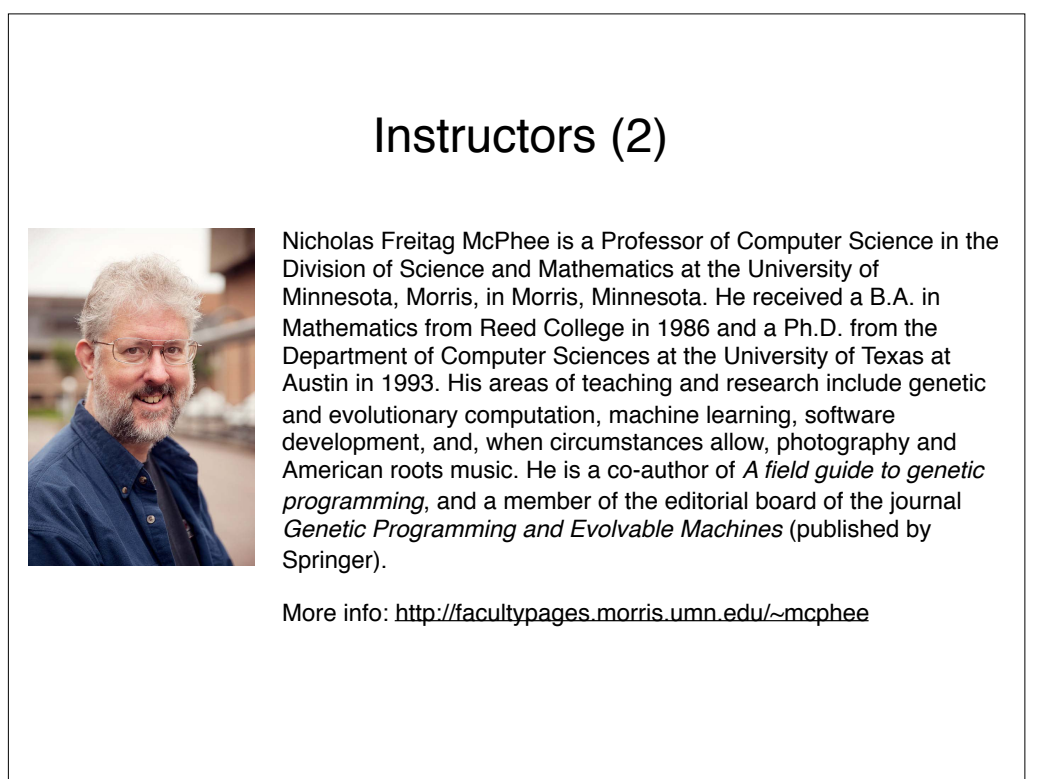

\title{
Outline
}

- The Push programming language

- Types and control without syntax

- Evolving Push programs

- Understanding expressive program evolution

- Evolving program evolution 


\section{Push}

- Programming language for programs that evolve

- Data flows via per-type stacks, not syntax

- Trivial syntax, rich data and control structures

- PushGP: GP system that evolves Push programs

- C++, Clojure, Common Lisp, Elixir, Java, Javascript, Python, Racket, Ruby, Scala, Scheme,

Swift; build your own in any language quickly

- http://pushlanguage.org

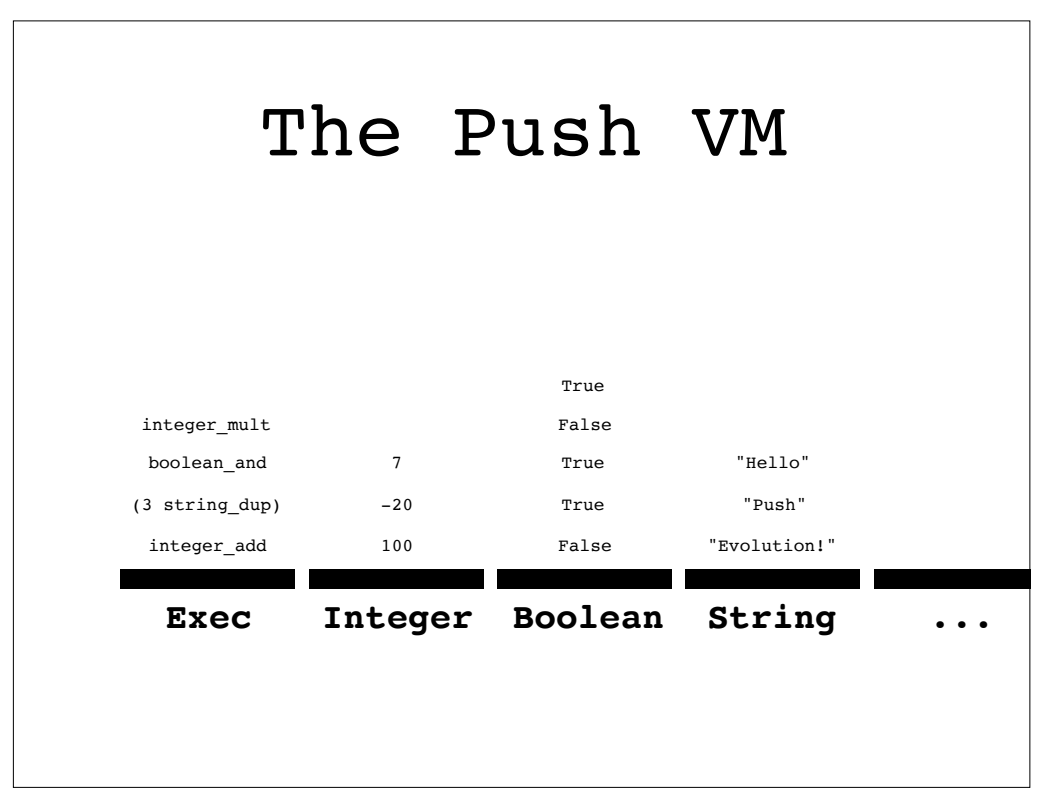

\section{Expressive}

- Multiple types

- Arbitrary control

- Multiple tasks (use lexicase selection)

- Self reproduction/variation (optional)

- Flexibility, resilience, neutrality

\section{Push Execution}

- Push the program onto the exec stack.

- While exec isn't empty and we haven't hit the step limit, pop and do the top:

- If it's an instruction, execute it. (Insufficient arguments? Do nothing.)

- If it's a literal, push it onto the appropriate stack.

- If it's a list, push its elements back onto the exec stack one at a time. 

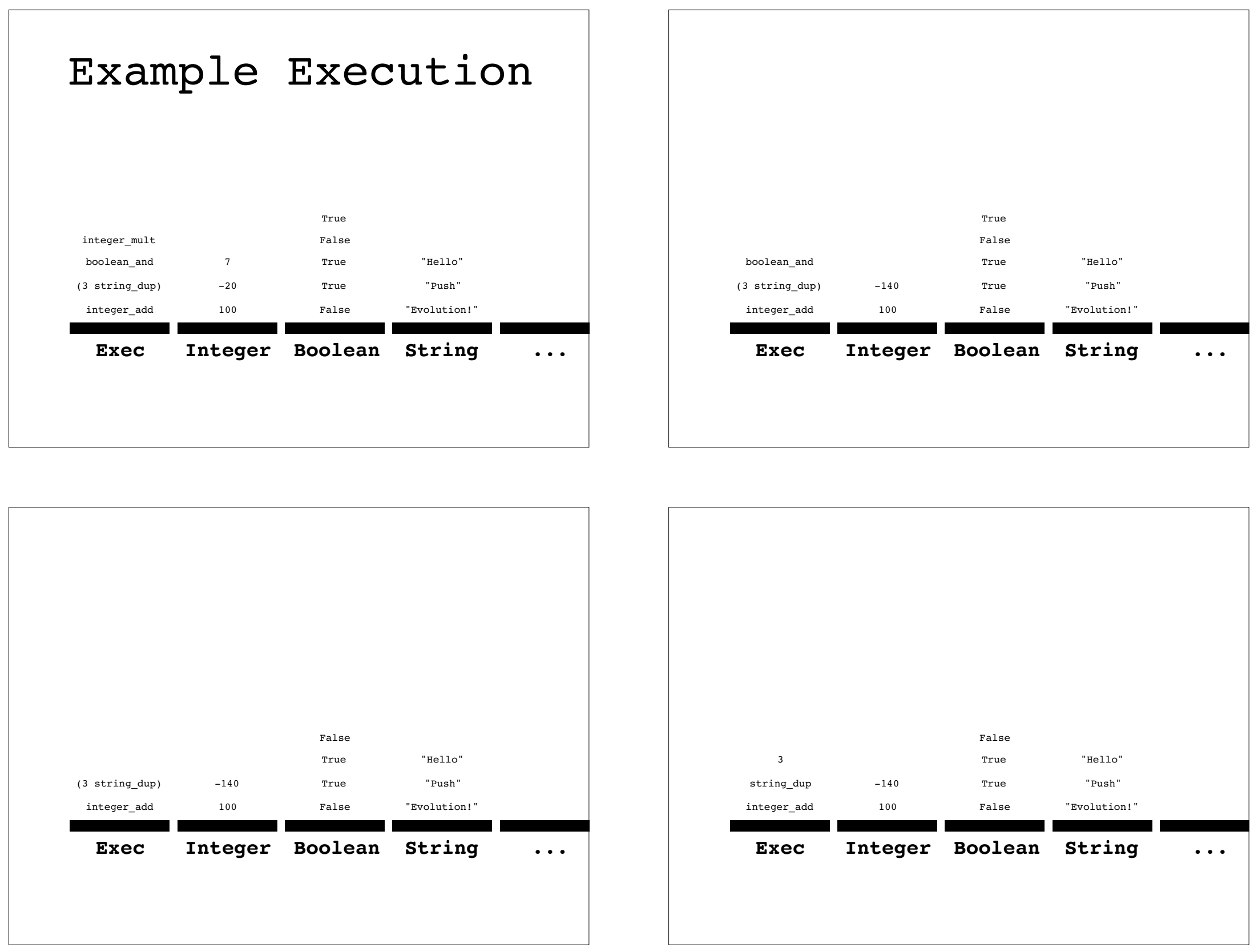

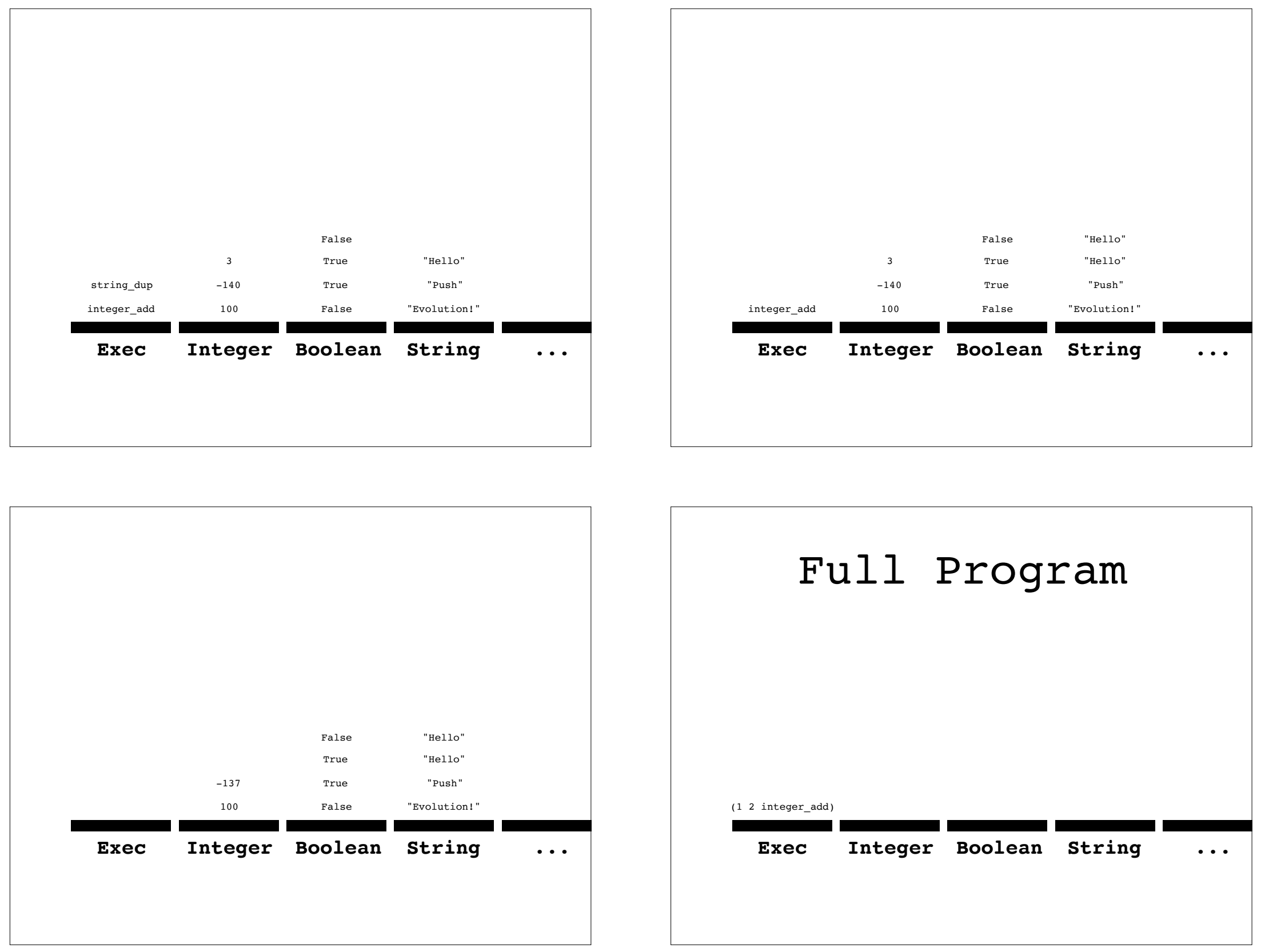

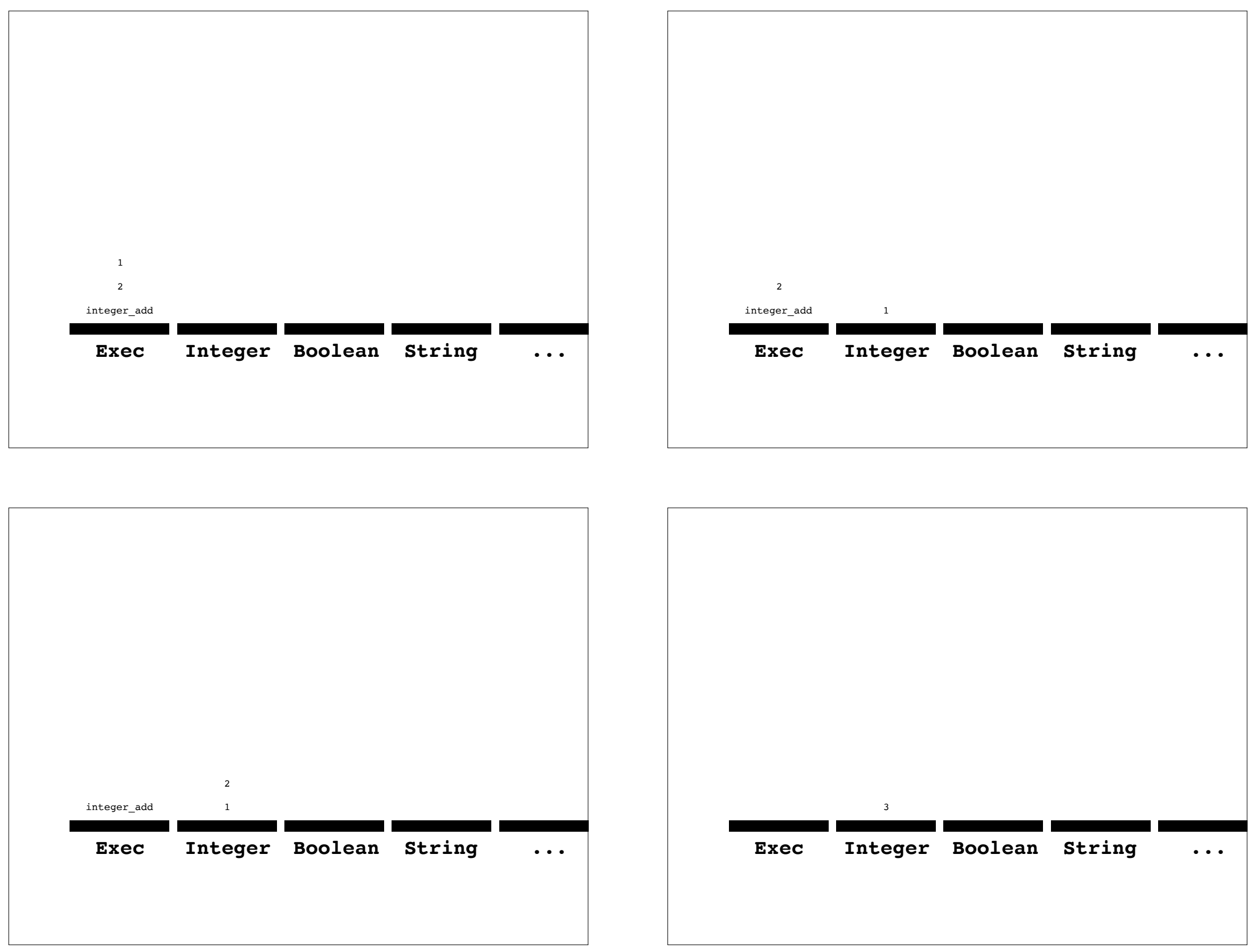
( 1 2 integer_add)

leaves 3 on the integer stack

(True False boolean_or boolean_not)

leaves False on the boolean stack

(3 5 integer_lte)

leaves True on the boolean stack

(3 5 integer_lte exec_if (1 "yes") (2 "no"))

leaves "yes" on string, 1 on integer

\section{For Most Types}

- <type>_dup

- <type> empty

- <type>_eq

- <type> flush

- <type>_pop

- <type> rot

- <type>-shove

- <type> stackdepth

- <type>_swap

- <type>_yank

- <type>_yankdup

\section{Selected Integer Instructions}

integer add integer dec integer div integer_gt integer_fromstring integer_min

integer_mult integer_rand

\section{Selected Boolean Instructions}

boolean_and boolean_xor boolean_frominteger

\section{Selected String Instructions}

string_concat string_contains string_length string_removechar string_replacechar

\section{Exec (selected)}

\section{Conditionals :}

exec_if exec_when

\section{General loops:}

exec_do*while

"For" loops:

exec_do*range exec_do*times

\section{Looping over structures:}

exec_do*vector_integer exec_string_iterate

\section{Combinators :}

exec_k exec_y exec_s 

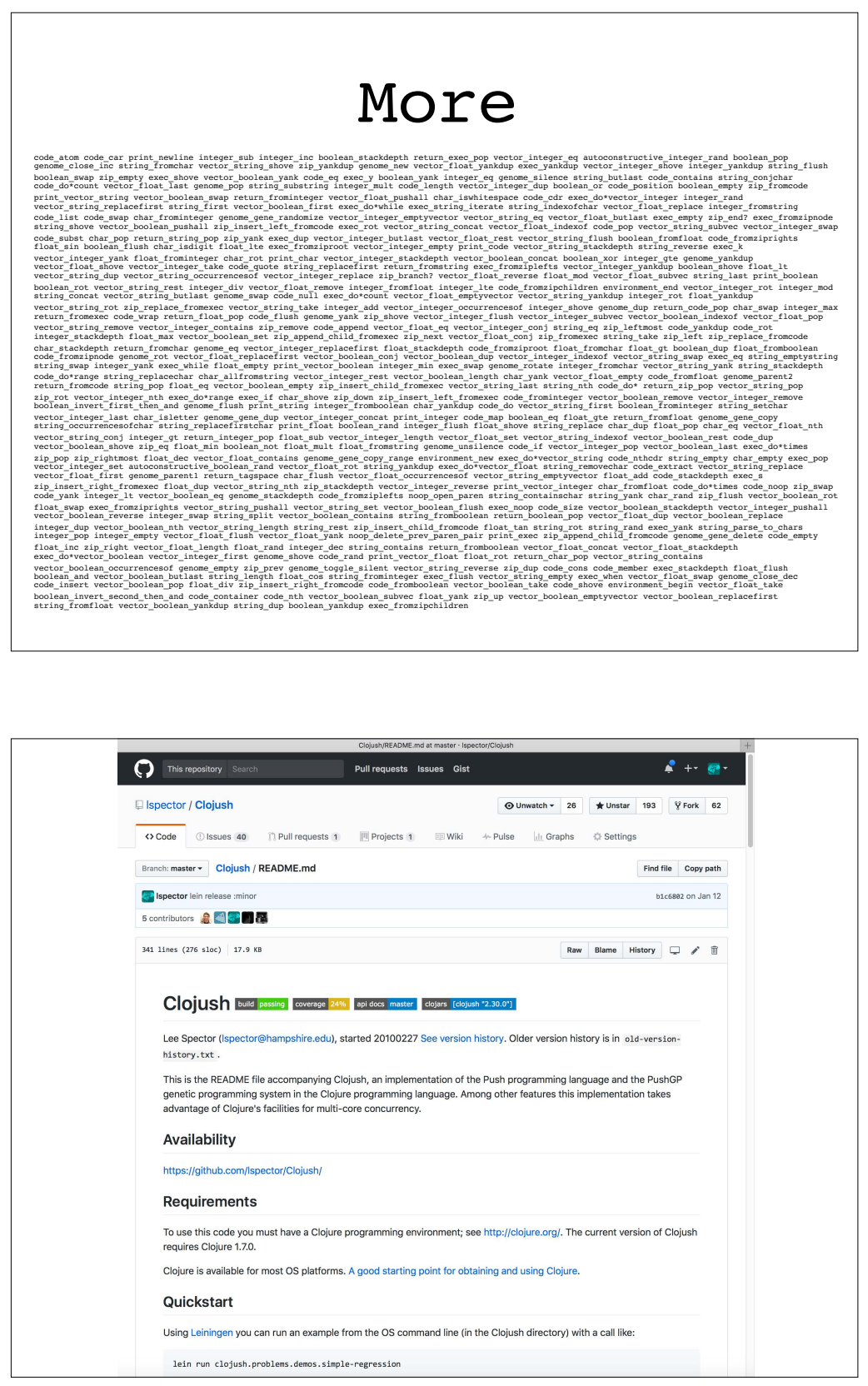

\section{More}

- Input instructions

- Print instructions

- Associative storage via tags

- Environment/return stacks

- Limits, termination modes

;; https://github.com/lspector/Clojush/

$\Rightarrow$ (run-push ' ( 12 integer add) (make-push-state)

: exec (( 12 integer_add $))$

: integer ()

:exec ( 12 integer_add)

:integer ( )

:exec ( 2 integer_add)

:integer (1)

: exec (integer_add)

: integer ( 2 1)

: exec ()

: integer (3) 


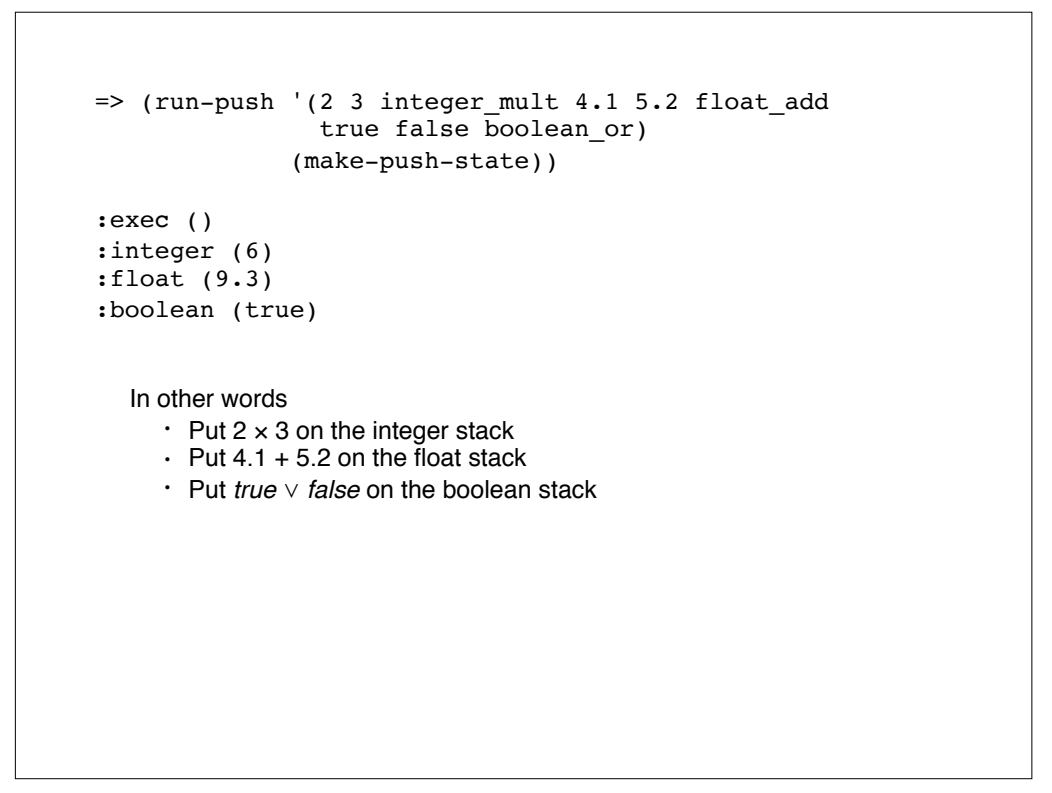

$=>$ (run-push

( 4.0 exec_dup ( 3.13 float_mult) 10.0 float_div) (make-push-state))

:exec (( 4.0 exec_dup $(3.13$ float_mult $) 10.0$ float_div $))$ : float ()

:exec ( 4.0 exec_dup ( 3.13 float_mult) 10.0 float_div) : float ()

:exec (exec_dup (3.13 float_mult) 10.0 float_div) : float $(4.0)$

:exec((3.13 float_mult) ( 3.13 float_mult) 10.0 float_div) :float (4.0)

$\cdots$

:exec ()

Computes $4.0 \times 3.13 \times 3.13 / 10.0$
$\Rightarrow$ (run-push ' ( 2 boolean_and 4.1 true integer_div false 35.2 boolean_or integer_mult float_add)

(make-push-state))

: exec (

: integer $(6)$

:float $(9.3)$

:boolean (true)

Same as before, but

Several operations (e.g., boolean_and) become NOOPs Interleaved operations

=> (run-push '( 18 exec do*range integer mult) (make-push-state))

: integer (40320)

Computes 8 ! in a fairly "human" way 
$=>$ (run-push ' (code_quote

(code_quote

code quote (code dup integer_dup

1 integer_sub code_

nteger_mult)

code_dup

intere if

code

(make-push-state)

: code ( (code quote (integer pop 1) code quote (code dup integer dup 1 integer sub code do integer mult) integer dup 2 integer lt code if) :integer $(4032 \overline{0})$

A less "obvious" recursive calculation of 8 ! achieved by code duplication

$\Rightarrow$ (run-push ' (in1 in1 float mult 3.141592 float mult) (push-item 2.5 :input (make-push-stäte)))

:float $(19.63495$

:input $(2.5)$

Computes the area of a circle with the given radius: $3.141592 \times$ in $1 \times$ in 1
$=>$ (run-push ' 0 true exec_while

( 1 integer_add true)

(make-push-state))

:exec ( 1 integer add true exec while ( 1 integer add true))

termination : abnormal

An infinite loop

Terminated by eval limit

Result taken from appropriate stack(s) upon termination

\section{Genetic Programming}

Random

Programs

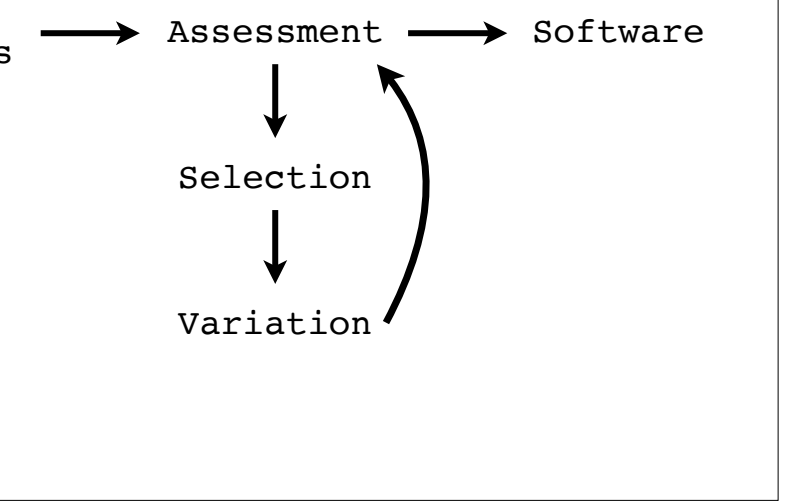




\section{Linear Genomes}

- Support uniform variation

- Structure where we want it, via translation

- ULTRA: Uniform Linear Transformation with Repair and Alternation

- PLUSH: epigenetic markers (Clojush)

- Plushy: close instructions (plushi, propel)

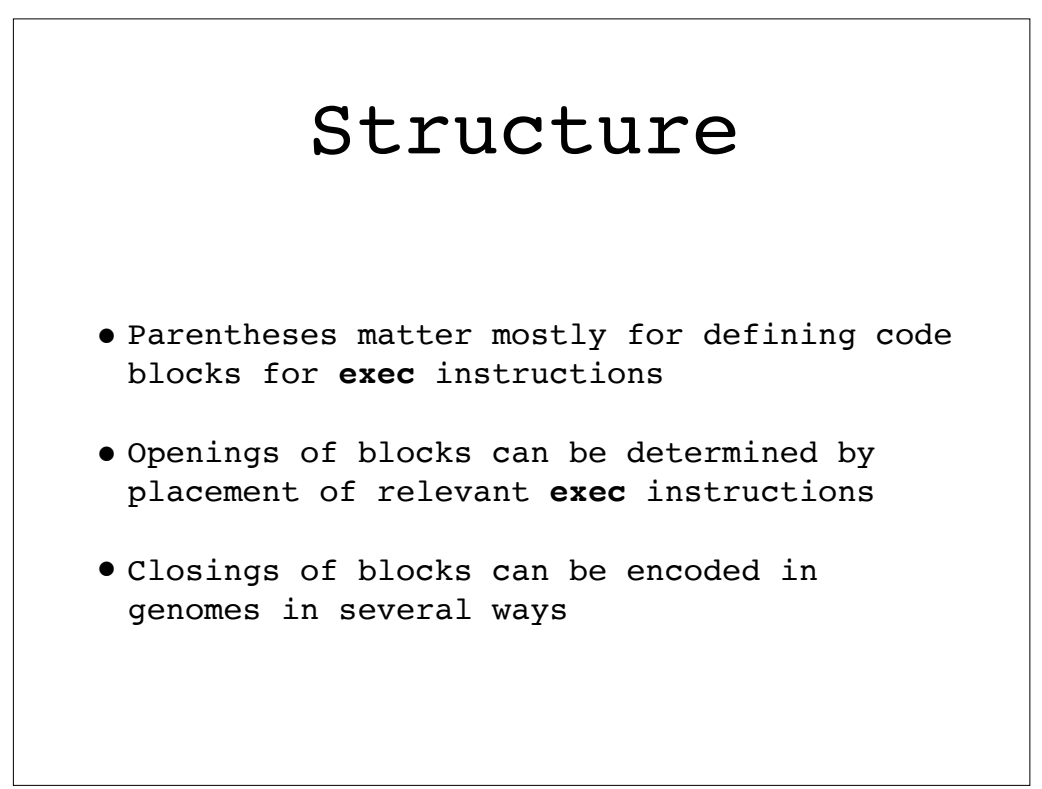

\section{Uniform Variation}

- All genetic material that a child inherits should be $\approx$ likely to be mutated

- Parts of both parents should be $\approx$ likely to appear in children (at least if they are $\approx$ in size), and to appear in a range of combinations

- Should be applicable to genomes of varying size and structure

\section{ULTRA}

- Linearize just during variation:

- Linearize two parents

- Alternate according to alternation rate, subject to alignment deviation

- Uniformly mutate, subject to mutation rate

- Repair

- Uniform, but structure anywhere 


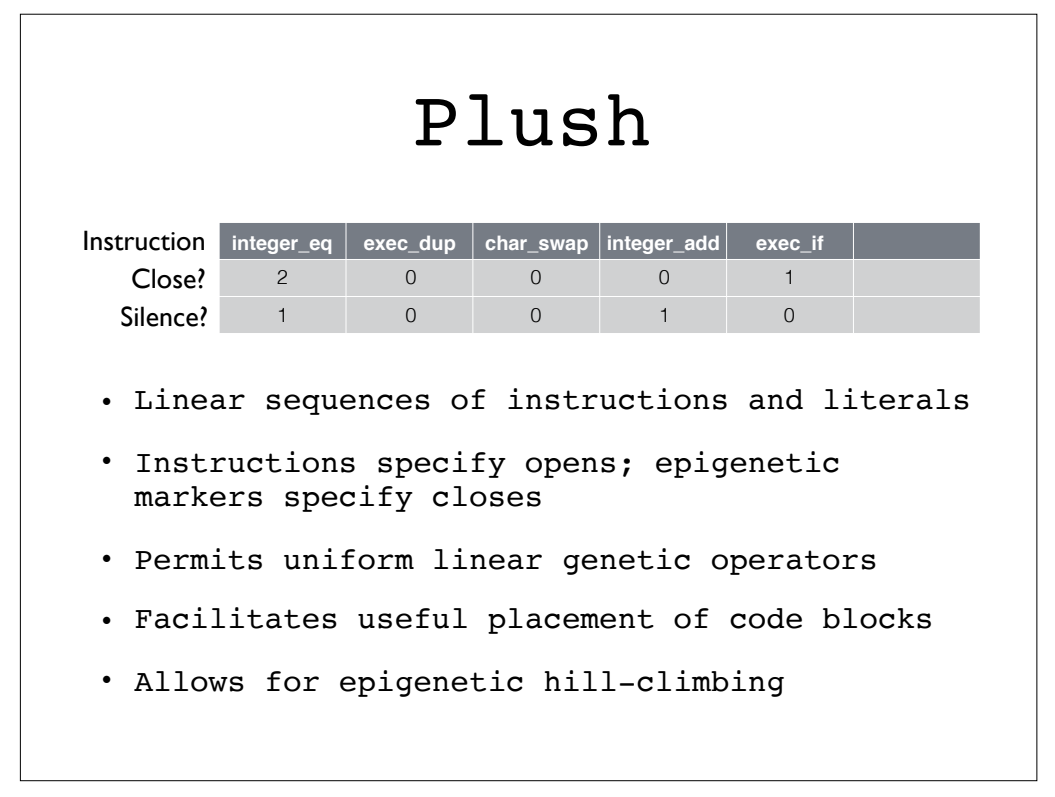

\section{Genetic Operators}

- Uniform mutation

- Alternation

- Uniform crossover

- Uniform mutation by addition and deletion (UMAD )

- Autoconstruction (genome instructions)

\section{Plushy}

- Instructions specify opens

- "close" pseudo-instructions specify closes

- Used in plushi, propel

\section{PushgP in Clojush}

\section{(pushgp}

Set up run for target $\mathrm{x}^{3}-2 \mathrm{x}^{2}-\mathrm{x}$

:error-function

(fn [ $\{$ : keys [program] :as individual $\}]$

(assoc individual :errors

(vec

(for [input (mapv float (range 10))]

(let [output ( $\rightarrow>$ (make-push-state)

(push-item input : input)

(run-push program)

(top-item : float))

(if (number? output)

(Math/abs (float (- output

(- (* input input input)

(* 2 input input)

1000000)1)1)1)

:atom-generators

'(in1 float_div float_mult float_add float_sub)\}) 


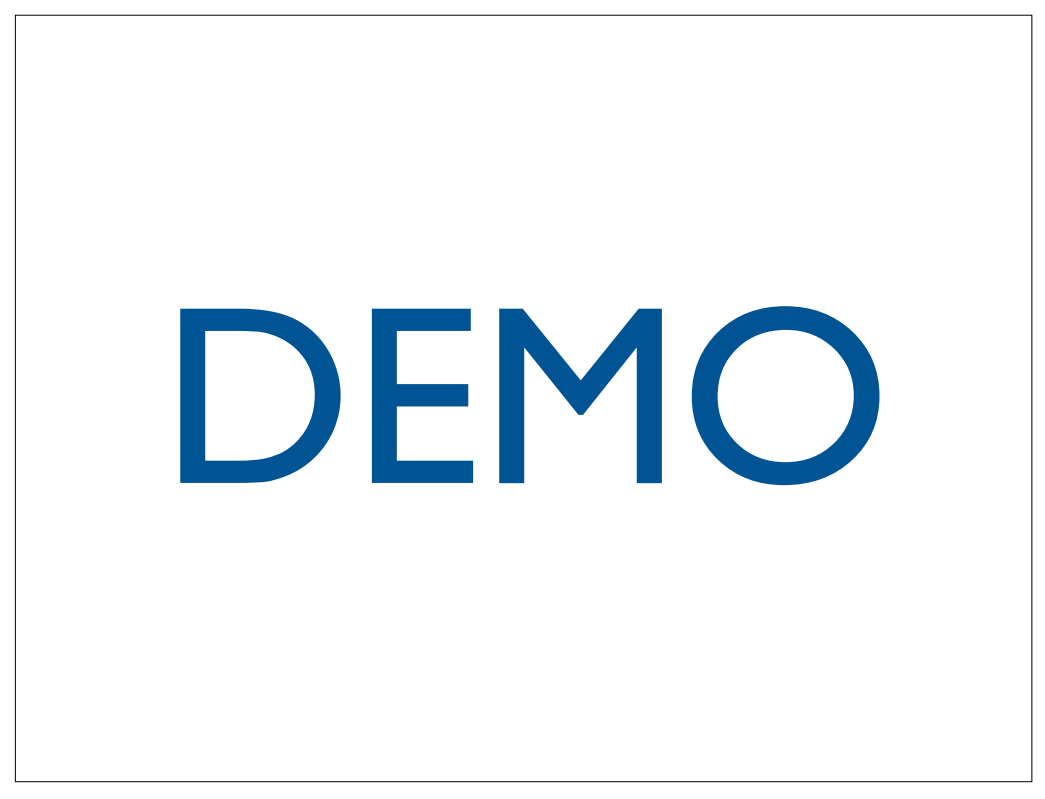

Problems Solved by PushgP in the GECCO-2005 Paper on Push3

- Reversing a list

- Factorial (many algorithms)

- Fibonacci (many algorithms)

- Parity (any size input)

- Exponentiation

- Sorting
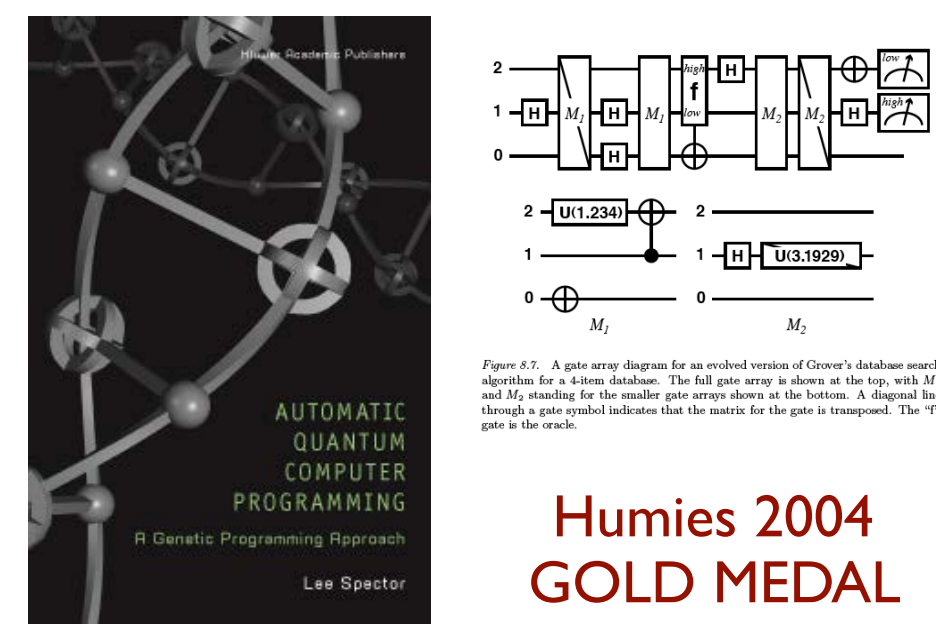

$2-U(1.234) \emptyset$

$1-\mathrm{H}, \overline{\mathrm{U}(3.1929)}$

$0-1$

0

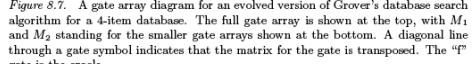

Humies 2004 GOLD MEDAL

\section{Genetic Programming for Finite Algebras}

Lee Spector
Cognitive Science

Hampshire College

Ispector@hampshire.edu

$\begin{array}{cc}\text { David M. Clark } & \text { lan Lindsay } \\ \text { Mathematics } & \text { Hampshire College } \\ \text { SUNY New Paltz } & \text { Amherst, MA 01002 } \\ \text { New PYltz, NY } 12561 & \text { iml04@hampshire.edu } \\ \text { clarkd@newpaltz.edu } & \\ \text { Barr } & \text { Jon Klein } \\ \text { Bollege } & \text { Hampsire College } \\ \text { 01002 } & \text { Amherst, MA 01002 } \\ \text { gmail.com } & \text { jk@artificial.com }\end{array}$

Humies 2008 GOLD MEDAL 


\section{Software Synthesis Benchmarks}

- Number IO, Small or Large, For Loop Index, Compare String Lengths, Double Letters, Collatz Numbers, Replace Space with Newline, String Differences, Even Squares, Wallis Pi, String Lengths Backwards, Last Index of Zero, Vector Average, Count Odds, Mirror Image, Super Anagrams, Sum of Squares, Vectors Summed, $\mathrm{X}$-Word Lines, Pig Latin, Negative to Zero, Scrabble Score, Word Stats, Checksum, Digits, Grade, Median, Smallest, Syllables

- PushGP has solved all of these except for the ones in blue

- Presented in a GECCO-2015 GP track paper

SUCCESS at generation 20

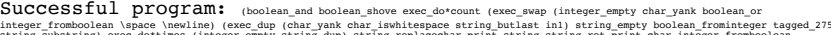

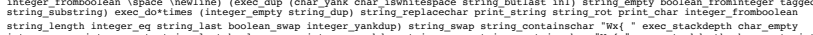

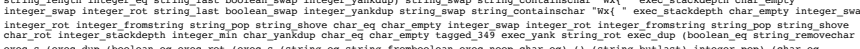

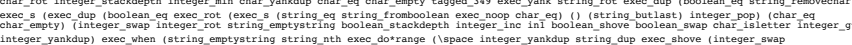

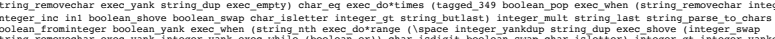

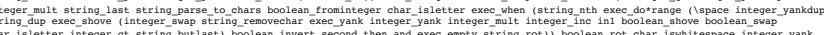

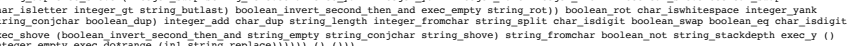
Errors: 1000

Total error: 0.0

Size: 23

Auto-simplifying with starting size: 231

step: 5000

program: ( \space \newline in1 string_replacechar print_string "Wx\{

string_last in1 string_removechar string_length)

errors: $100000000000000000000000000000000000000000 \ldots 0000000000000$

Total error: 0.0

Size: 11

\section{Auto-Simplification}

\section{- Loop:}

- Make it randomly simpler

- Keep simpler if as good or better; otherwise revert

- GECCO-2014 poster: efficiently and reliably reduces the size of the evolved programs

- GECCO-2014 student paper: used as genetic operator

- GECCO-2017 GP best paper nominee: improves generalization

\section{Checksum}

Multiple types, looping, and code blocks

Simplified solution:

( "Check sum is " print_string

in1 64 exec_string_iterate (integer_fromchar integer_add)

64 integer_mod

Ispace integer_fromchar integer_add char_frominteger print_char) 

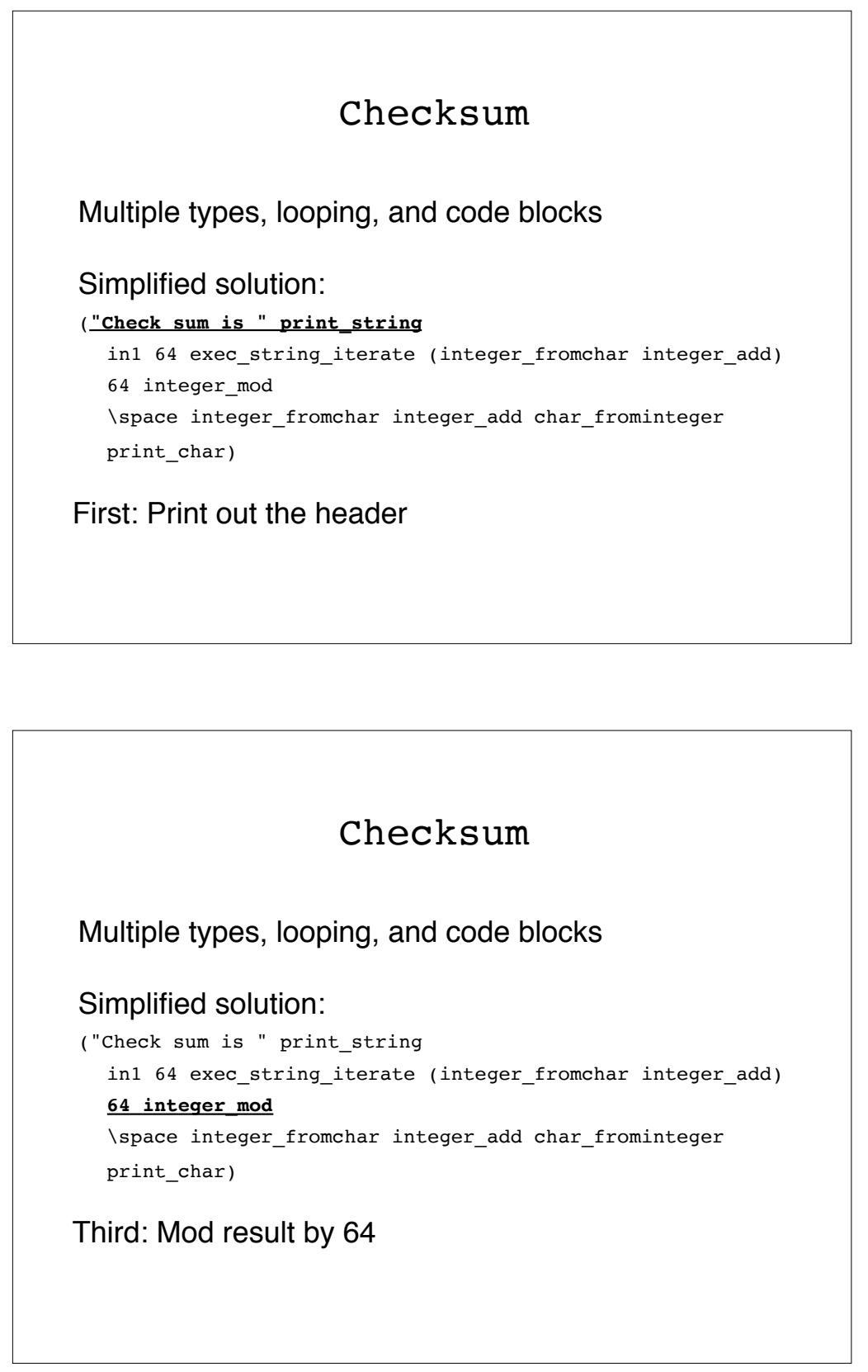

\section{Checksum}

Multiple types, looping, and code blocks

\section{Simplified solution:}

("Check sum is " print_string

in1 64 exec_string_iterate (integer_fromchar integer_add)

64 integer_mod

(space integer_fromchar integer_add char_frominteger print_char)

Second: Convert each character to an integer, sum, and add to 64 .

\section{Checksum}

Multiple types, looping, and code blocks

\section{Simplified solution:}

("Check sum is " print_string

in1 64 exec_string_iterate (integer_fromchar integer_add)

64 integer_mod

Ispace integer fromchar integer add char frominteger print_char)

Third: Add modulus result to 32 and convert to char 

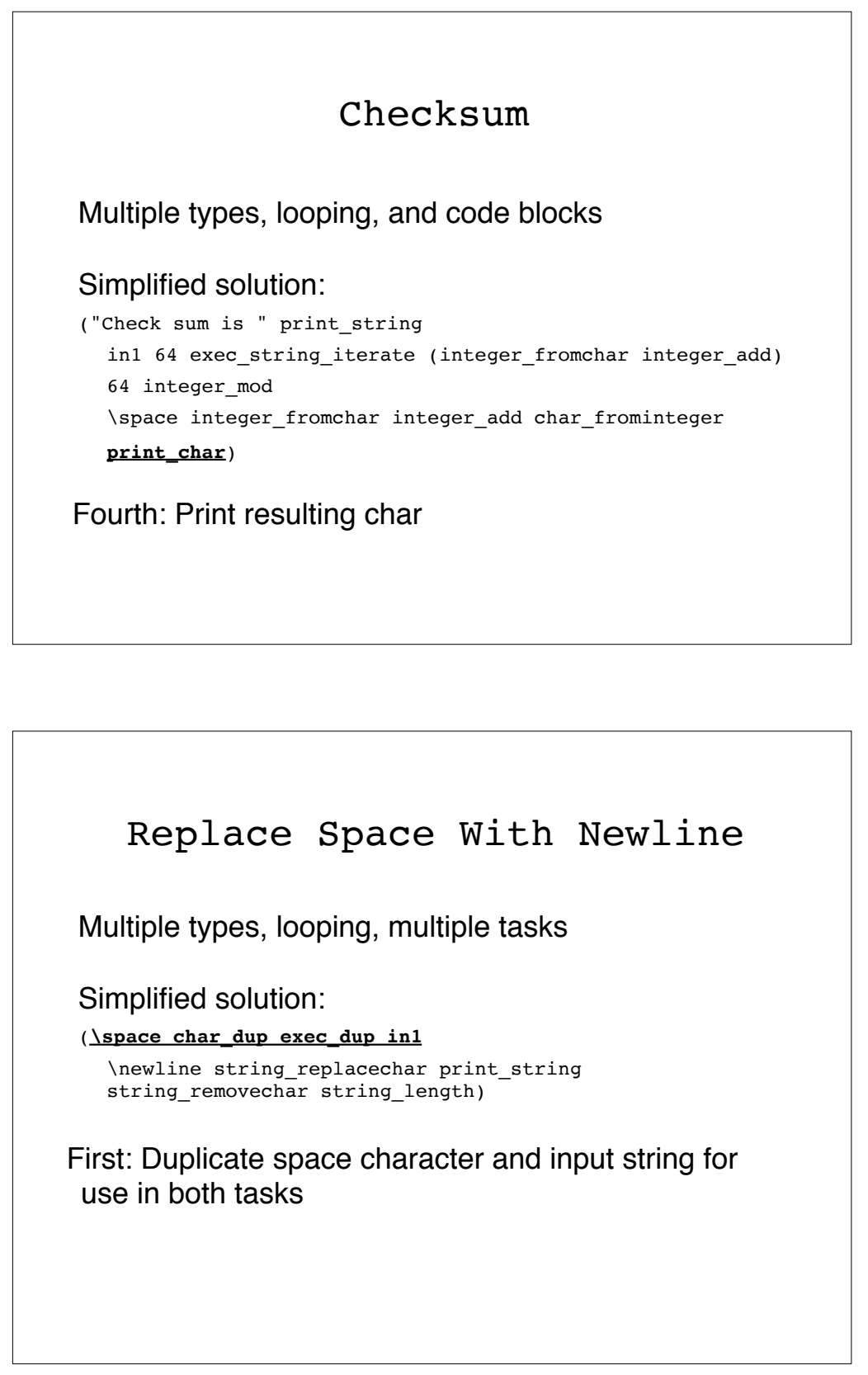

\section{Replace Space With Newline}

Multiple types, looping, multiple tasks

Simplified solution:

( \space char_dup exec_dup in1

Inewline string_replacechar print_string string_removechar string_length)

\section{Replace Space With Newline}

Multiple types, looping, multiple tasks

Simplified solution:

(Ispace char_dup exec_dup in1

Inewline string replacechar print string

string_removechar string_length)

Second: Replace spaces with newlines and print 


\section{Replace Space With Newline}

Multiple types, looping, multiple tasks

\section{Simplified solution:}

(Ispace char_dup exec_dup in1

Inewline string_replacechar print_string

string removechar string length)

Third: Remove all spaces from second copy of input, and push length of result on integer stack for return

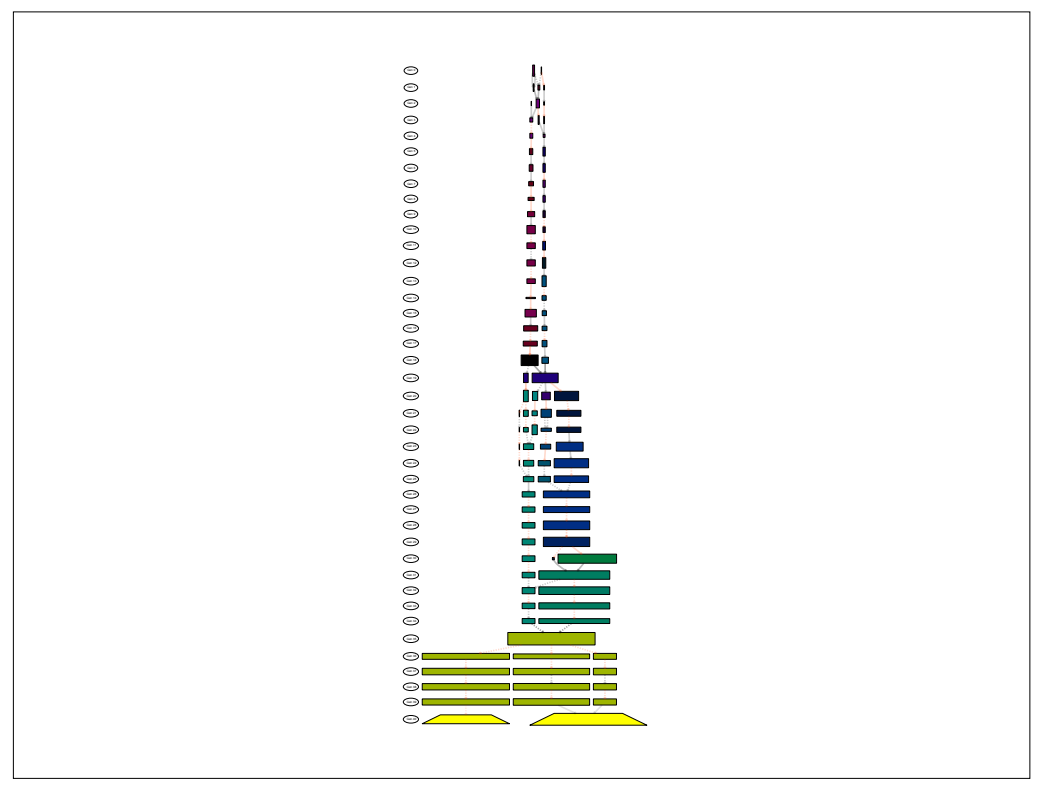

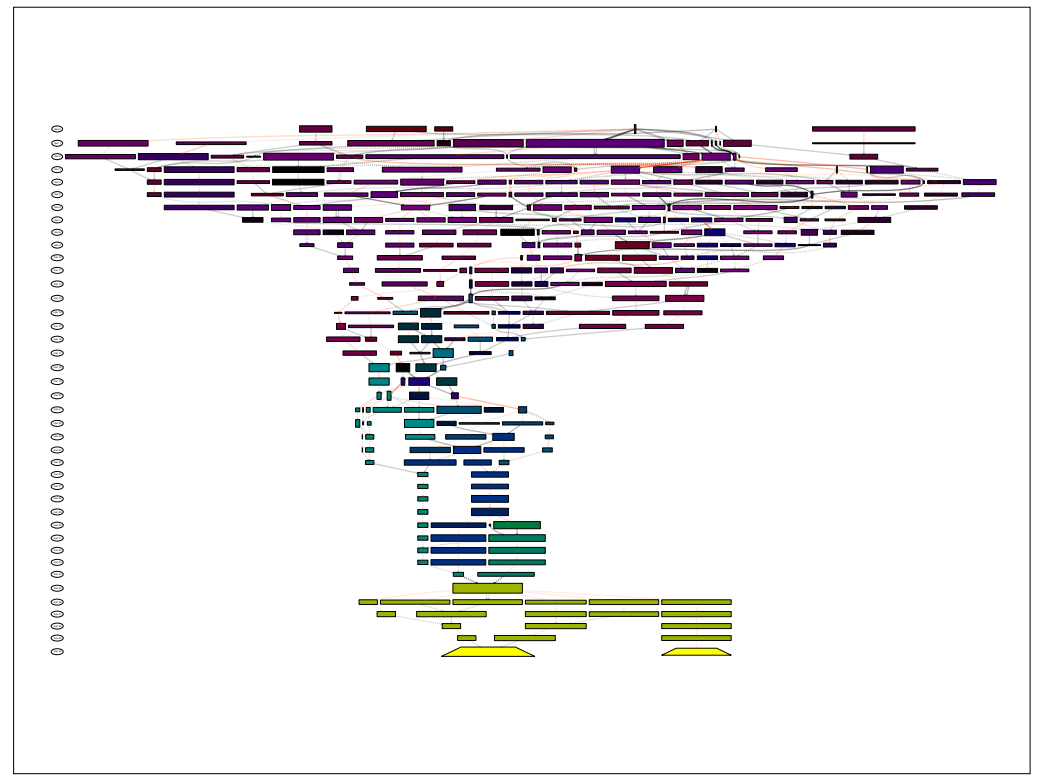

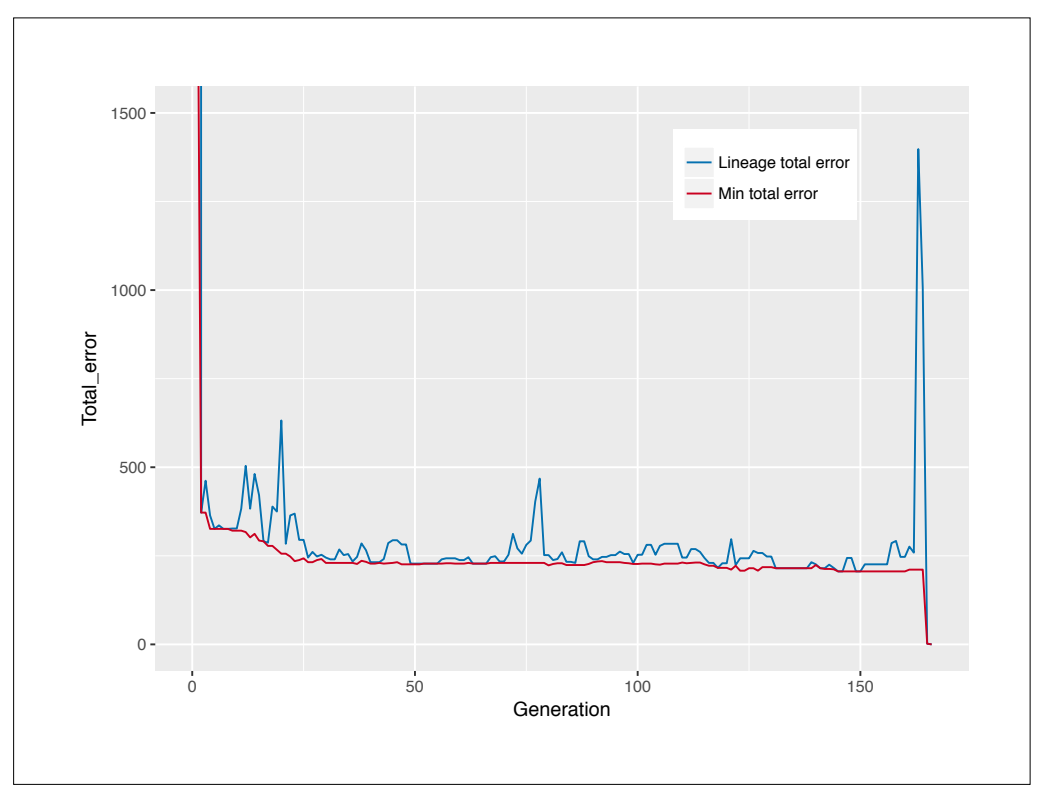



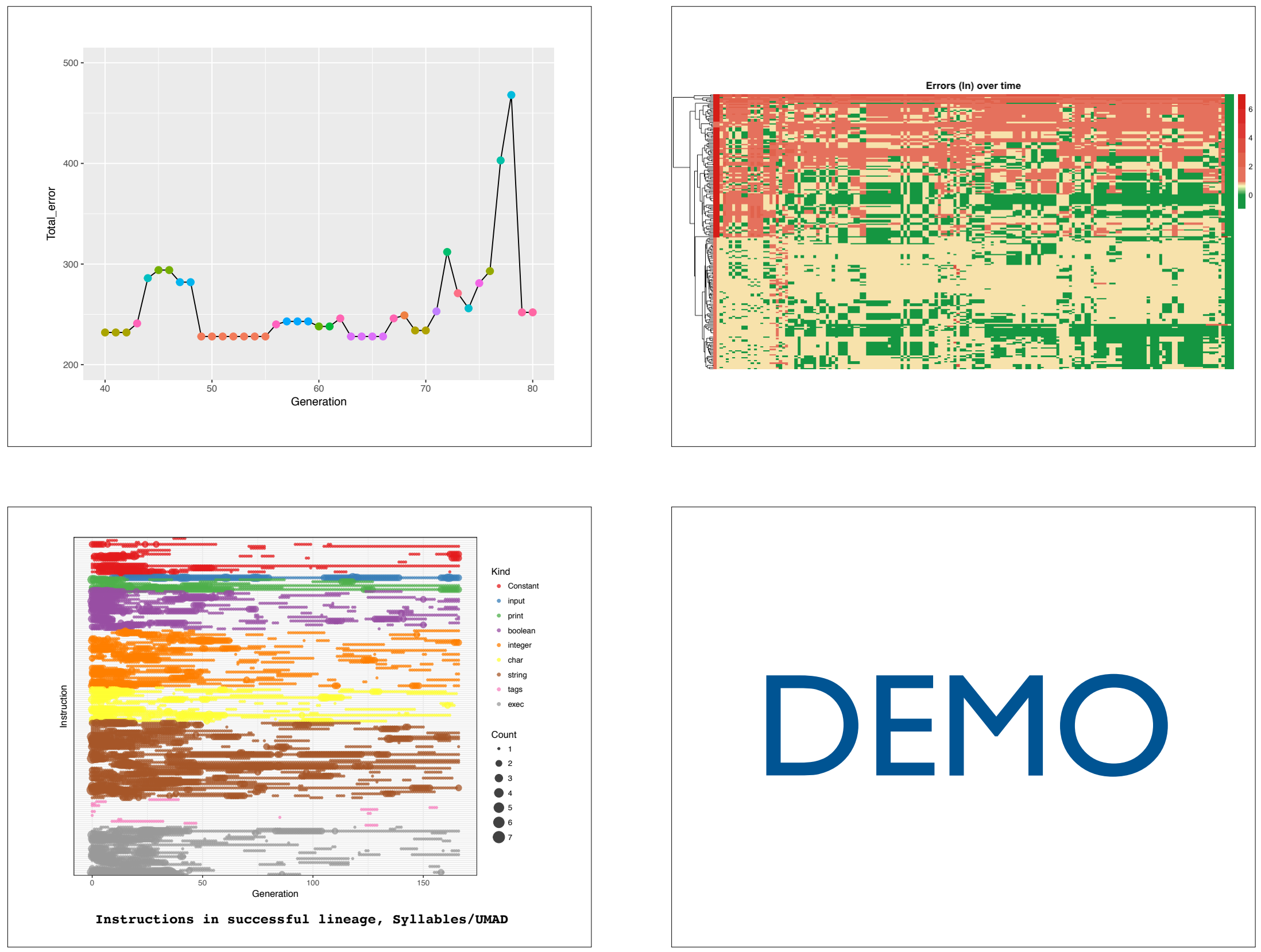
Variation in GP

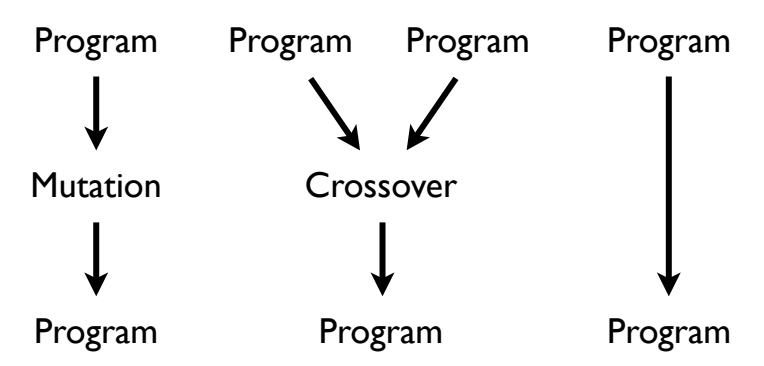

Variation in GP

Written and configured by humans

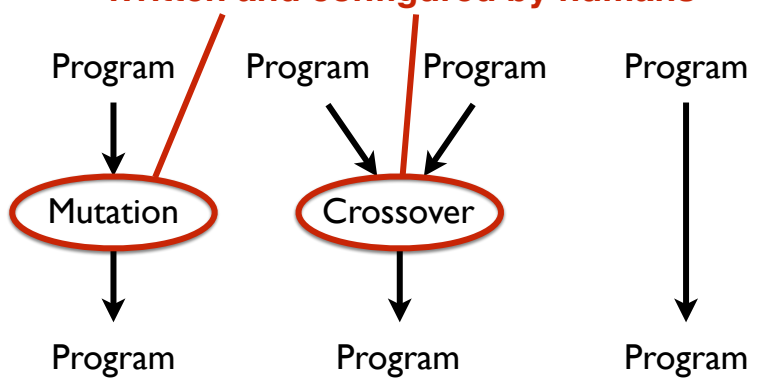

Autoconstruction

Program

Execute!

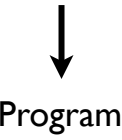

Autoconstruction

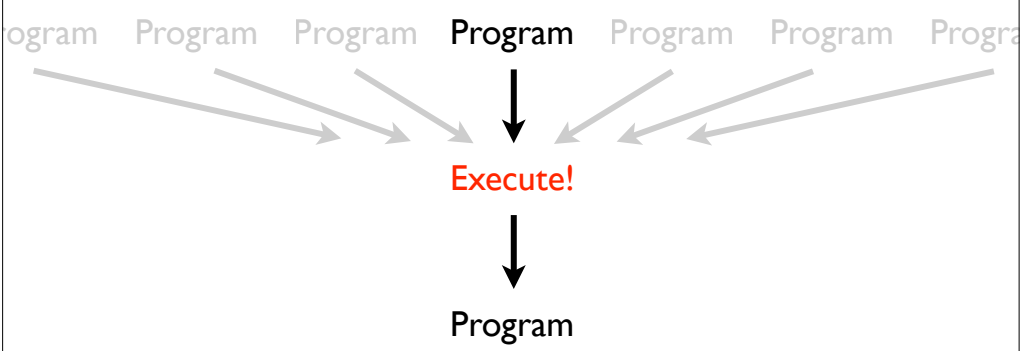

A bit more complicated when genomes distinguished from programs

994 


\section{Autoconstruction}

- Evolve evolution while evolving solutions

- How? Individuals produce and vary their own children, with methods that are subject to variation

- Requires understanding the evolution of variation

- Hope: May produce EC systems more powerful than we can write by hand

\section{For Evolution²}

- Diversity: Individuals vary

- Diversification: Individuals produce descendants that vary, in various ways

- Recursive Variance: Individuals produce descendants that vary in the ways that they vary their offspring

\section{Autoconstruction}

- A 15 year old project (building on older and broader-based ideas)

- Like genetic programming, but harder and less successful! But with greater potential?

- Recent versions sometimes solve significant problems, intriguing patterns of evolving evolution

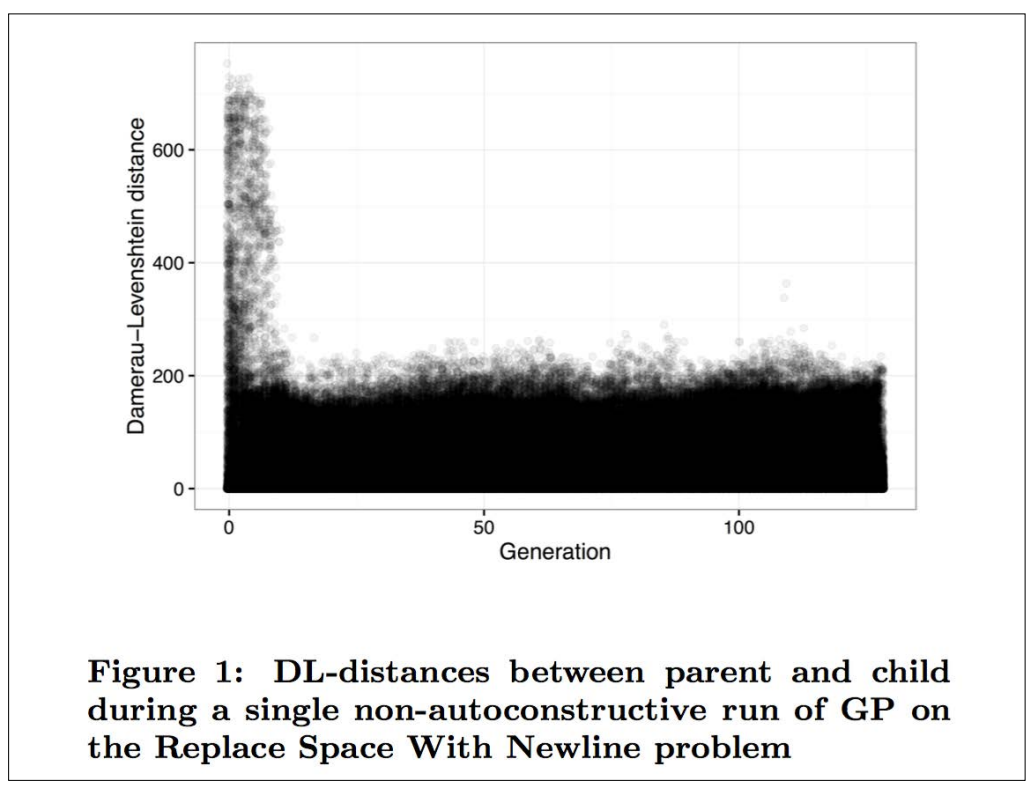




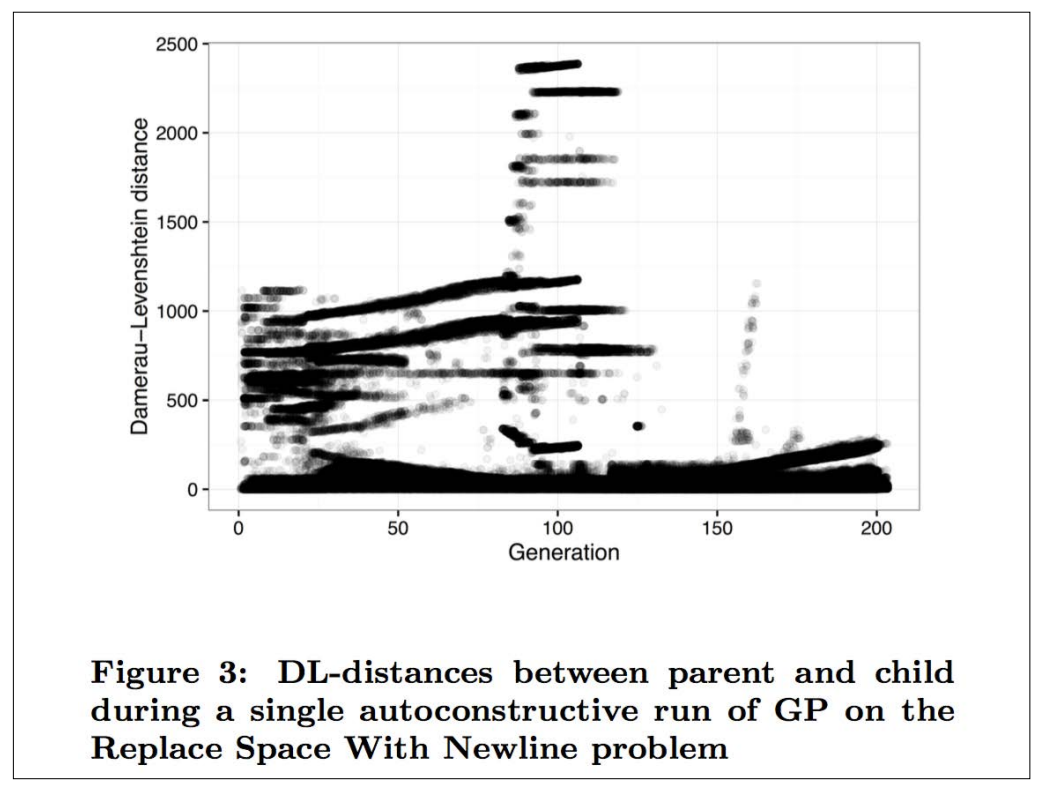

\section{Conclusions}

- Push supports evolution of expressive programs that use arbitrary types and control structures, possibly to perform multiple tasks

- Push interpreters, and GP systems that evolve Push programs, are easy to write

- Push supports research on expressiveness in genetic programming, for example to support the evolution of modularity

\section{Evolution Evolving}

- Autoconstructive evolution can sometimes succeed as much and as fast as non-autoconstructive evolution

- Autoconstruction found solutions for the string differences software synthesis problem before ordinary GP

\section{Thanks}

Thanks to the members of the Hampshire College Computational Intelligence Lab, to Josiah Erikson for systems support, and to Hampshire College for support for the Hampshire College Institute for Computational Intelligence.

This material is based upon work supported by the National Science Foundation under Grant No. 1617087. Any opinions, findings, and conclusions or recommendations expressed in this publication are those of the authors and do not necessarily reflect the views of the National Science Foundation. 


\section{References}

The Push language website: http://pushlanguage.org

Helmuth, Thomas, Nicholas Freitag McPhee, and Lee Spector. 2018. Program Synthesis using Uniform Helmuth, Thomas, Nicholas Freitag McPhee, Edward Pantridge, and Lee Spector. 2017. Improving
Generalization of Evolved Programs through Automatic Simplification. In Proc. Genetic and Evolution Computation Conference. ACM Press.

Helmuth, Thomas, Lee Spector, Nicholas Freitag McPhee, and Saul Shanabrook. Linear Genomes for Structured Programs. In Worzel, William, William Tozier, Brian W. Goldman, and Rick Riolo, Eds., Gene Programming Theory and Practice XIV. New York: Springer. McPhee, Nicholas Freitag, Mitchell D. Finzel, Maggie M. Casale, Thomas Helmuth and Lee Spector. A
detailed analysis of a PushGP run. In Worzel, William. William Tozier, Brian W. Goldman, and Rick Riole,

Spector, L., N. F. McPhee, T. Helmuth, M. M. Casale, and J. Oks. 2016. Evolution Evolves wi ho

ACM Press. pp. 1349-1356.

Evolutionary Computation Conference. ACM Press. pp. 1039-1046.

La Cava, W., and L. Spector. 2015. Inheritable Epigenetics in Genetic Programming. In Genetic

La Cava, W., and L. Spector. 2015. Inhertable Epigenetics in Genetic
Programming Theory and Practice XII. New York: Sppringer. pp. $37-51$.

Helmuth, T., L. Spector, and J. Matheson. 2015. Solving Uncompromising Problems with Lexicase Selection.

Helmuth, T., L. Spector, and J. Matheson. 2015. Solving Uncompromisisig
In IEEE Transactions on Evolutionary Computation 19(5), pp. 630-643.
Helmuth, T., and L. Spector. 2014. Word Count as a Traditional Programming Benchmark Problem for Genetic Spector, L., and T. Helmuth. 2014. Eirective Simplification of Evolved Push Programs Using a Simple, Stochastic Hill-climber. In Companion
Conference. ACM Press. pp. 147-148.

Zhan, H. 2014. A quantitative analysis of the simplification genetic operator. In Companion Publication of the
2014 Genetic and Evolutionary Computation Conference ACM Press ACM Press. pp. 1077-1080.

Eelmuth. 2012. Tag-based Modularity in Tree-based Genetic Programming. In Proc. Genetic and Evolutionary Computation Conference. ACM Press. pp. 815-822.

Spector, L., K. Harrington, B. Martin, and T. Helmuth. 2011. What's in an Evolved Name? The Evolution of Modularity via Tag-Based Reference. In Genetic Programming Theory and Practice IX. New York: Springer. pp.

Spector, L. 2010. Towards Practical Autoconstructive Evolution: Self-Evolution of Problem-Solving Genetic
Programming Systems. In Genetic Programming Theory and Practice VIII, R. L Riolo, T. McConaghy, and E. Vladislavleva, eds. Springer. pp. 17-33.

Spector, L., D. M. Clark, I. Lindsay, B. Barr, and J. Klein. 2008. Genetic Programming for Finite Algebras. In Spector, L., J. Klein, and M. Keijzer. 2005. The Push3 Execution Stack and the Evolution of Control. In Proc
Genetic and Evolutionary Computation Conference. Springer-Verlag. pp. 1689-1696. Spector, L. 2004. Automatic Quantum Computer Programming: A Genetic Programming Approach. Boston, MA: Kluwer Academic Publishers.

Spector, L., and A. Robinson. 2002. Genetic Programming and Autoconstructive Evolution with the Push Programming Language. In Genetic Programming and Evolvable Machines, Vol. 3, No. 1, pp. 7-40. Spector, L. 2001. Autoconstructive Evolution: Push, PushGP, and Pushpop. In Proc. Genetic and Evolutionary Computation Conference. Morgan Kautmann Publishers. pp. 137-146.

Robinson, A. 2001. Genetic Programming: Theory, Implementation, and the Evolution of Unconstrained 